Annales Geophysicae (2001) 19: 933-944 (C) European Geophysical Society 2001

(5)

\title{
The structure of turbulence in the middle and lower atmosphere seen by and deduced from MF, HF and VHF radar, with special emphasis on small-scale features and anisotropy
}

\author{
W. K. Hocking ${ }^{1}$ and J. Röttger ${ }^{2}$ \\ ${ }^{1}$ Dept. of Physics and Astronomy, University of Western Ontario, London, Ont. N6A 3K7, Canada \\ ${ }^{2}$ Max-Planck-Institut für Aeronomie, Katlenburg-Lindau, Germany
}

Received: 23 October 2000 - Revised: 11 June 2001 - Accepted: 10 July 2001

\begin{abstract}
An overview of the turbulent structures seen by MF, HF and VHF radars in the troposphere, stratosphere and mesosphere is presented, drawing on evidence from previous radar measurements, in situ studies, laboratory observations, observations at frequencies other than those under focus, and modeling studies. We are particularly interested in structures at scales less than one radar pulse length, and smaller than the beam width, and especially the degree of anisotropy of turbulence at these scales. Previous radar observations are especially important in regard to the degree of anisotropy, and we highlight the role that these studies have had in furthering our understanding in this area. The contrasts and similarities between the models of anisotropic turbulence and specular reflection are considered. The need for more intense studies of anisotropy at MF, HF and VHF is especially highlighted, since this is an area in which these radars can make important contributions to the understanding of atmospheric turbulence.
\end{abstract}

Key words. Meteorology and atmospheric dynamics (turbulence) - Atmospheric composition and structure (instruments and techniques) - History of geophysics (atmospheric sciences)

\section{Introduction}

Radars depend on scatter or reflection from perturbations in radio refractive index in order for them to be able to function as tools for atmospheric studies. Turbulence, both in the neutral atmosphere and in ionized parts of the air, is one way by which such perturbations are produced. Our intention in this article is to develop a picture of the structure of turbulence as seen by MF, HF and VHF radars in the upper troposphere, the stratosphere and the mesosphere. We will draw our evidence from a variety of sources, especially concentrating on (but not restricting ourselves to) previous radar

Correspondence to: W. K. Hocking

(whocking@danlon.physics.uwo.ca) observations. Thus, the article serves not only as a way to describe the characteristics of turbulence as seen by radars at these frequencies, but also chronicles the contributions of these radars to understanding the small-scale structures inherent within the turbulence. We also recognize that an ongoing debate exists in regard to the relative contributions of turbulent scatter and specular reflection in VHF radar studies, and we will return to this issue later. For now, we simply recognize that turbulent scatter does exist at least some of the time, and in this article, we choose to focus for the most part on this type of scatter. We will discuss here only turbulence in the neutral atmosphere. Scatter from plasma turbulence and plasma waves in the ionosphere will not be covered we refer to Sahr and Fejer (1996) for a corresponding review of ionospheric turbulence.

Before beginning, we first need to define what we mean by the concept of turbulence within this article. We consider turbulence to be quasi-chaotic, non-linear, partly rotational atmospheric motions which produce inhomogeneities in density and temperature (and other parameters such as humidity and pressure) that lack a dominant coherent structure and which can best be described in terms of statistics and correlation functions. The quasi-random motions which ensue within a patch of turbulence, and the subsequent temperature and density (and other) variations produce refractive index inhomogeneities which are responsible for backscatter of radio waves. The refractive index perturbations can be represented as a spectrum, with certain critical scales defining the limits of the turbulence. Important scales include the Kolmogoroff microscale, which defines the regime where the scales are heavily damped due to viscous heat losses, and the buoyancy scale, which defines the typical scales at which the turbulence is generated. Turbulence is considered here to be generally the result of a non-linear breakdown of larger, more organized structures which have become unstable, with scales comparable to or larger than the buoyancy scale. Our definition of turbulence includes the cases of growing, steady-state and decaying turbulence, although we recognize that many theories of turbulence concentrate only 
on the steady-state.

When we talk here about scales of turbulence, we need to recognize that radar backscatter results from scales in the turbulence spectrum (which in turn determines the radio refractive index variation spectrum), which are equal to onehalf of the radar wavelength (the so-called Bragg scale). The backscattered power is the ensemble average over the total illuminated volume, often (but not always) consisting of a multitude of so-called "scatterers". Such scatterers can be quite arbitrarily and inhomogeneously distributed in the volume, and the volume filled by the scatterers is usually much larger than the Bragg scale. The radars cannot normally resolve turbulence structures at the Bragg scale itself, but they can sometimes resolve scales which are larger than the Bragg scale but smaller than the volume filled by the turbulence. The actual resolution is often defined by the radar pulse length, but on occasions, with suitable special techniques, it is possible to do better. These special techniques are noted in this article, together with the description of the radar-observed structures and their morphology.

Radars have been especially good for looking at the largerscale nature of atmospheric turbulence and particular coherent structures, and these results will be described here. However, despite the strong dependence of radar backscatter on turbulence, it has, in fact, been rather difficult to deduce certain other types of information about turbulence from these radar studies. This is especially true with respect to studies at very small scales, particularly at sizes less than the pulse length used by the radar. Therefore, many of the most useful studies have incorporated a mixture of special radar observations, in situ studies, and careful deduction.

\section{Large-scale studies $(>100 \mathrm{~m})$}

Perhaps the most obvious feature that is clear from radar studies is the horizontally striated nature of atmospheric turbulence, at least at scales of more than a few hundred metres. Figure 1a shows some radar observations of the mesosphere carried out by Czechowsky et al. (1979). The stratified nature of the scatter is quite evident; this is very common. For comparison, Fig. 1b (from Bondarev et al., 1992) shows some in situ smoke trails released simultaneously by 4 rockets into the atmosphere (in this case, the results refer to the stratosphere). The layering in the wind-field is quite evident, with adjacent trails very similar in shape. Figure $1 \mathrm{c}$ shows more radar observations of thin layers, also called sheets or laminae, in this case for the troposphere and lower stratosphere (Hocking and Röttger, 1983). It is clear that layered and stratified phenomena are common in the atmosphere. Similar results have been observed using rocket releases in the mesosphere (e.g. Blamont and Barat, 1967).

Radars have played an important role in demonstrating the prevalence of atmospheric stratification. When combined with suitable modeling studies, it is even possible to make some estimate of the expected degree of stratification and structure in the atmosphere. Figure $1 \mathrm{~b}$ shows not only a high level of stratification, but also evidence for wave-like structures in the wind-field. These are associated with a spectrum of gravity waves (e.g. Van Zandt, 1982; Hines, 1991a, b). The interactions between these waves, and with the background wind-field, are often responsible for the generation of turbulence in the mesosphere and stratosphere. (Indeed, radar studies of wind fluctuations played a very important role in establishing the importance of gravity waves in the atmosphere, but that topic is beyond the scope of this article.) If it is assumed that these waves satisfy a so-called "universal spectrum", and that there are many waves propagating up and down with various (random) phases, then the velocity and temperature fields associated with these waves will add in such a way that, on occasion, they will produce a regime in which turbulence will naturally develop (Richardson number less than 0.25 ). It is possible to produce a statistical investigation of the likelihood of such levels developing, and thereby obtain a statistical summary of the likely depths of these layers, their frequency of occurrence, and their likely vertical spacing. Figure 2 shows an example of such statistics (Hocking, 1991, after adaptation from Desaubies and Smith, 1982). Similar calculations have been performed by Fairall et al. (1991) for the troposphere, and by Hines (1991a, b, c) for the mesosphere.

Radars have not only been useful in helping us understand the distribution of turbulence, but have also been useful in helping to measure the energy dissipation rates associated with it (e.g. for reviews of these methods, which involve both absolute power measurements and spectral-width determinations, see Hocking and Mu, 1997; Hocking, 1999). Radars can also be used to parameterize the processes of diffusion in the atmosphere. While we will not consider this application in any detail here since the focus of the paper is related to the structure of turbulence, there is one aspect related to the issue of diffusion that we do wish to discuss, and this is the mechanism of diffusion itself, since it relates closely to the issue of the spatial and temporal morphology of turbulence in the atmosphere.

Particulates and chemicals may diffuse across a patch of turbulence in the classically accepted manner, being driven along a "random walk" by the velocity field embodied in the turbulence. However, this only permits counter-gradient diffusion across the layer itself. What about diffusion over vertical scales much deeper than a typical layer thickness? How can diffusion occur over such large scales? To understand this process, we must return to Fig. 2. Layers of the type shown in Fig. 2a form at various intervals of time, and have various lifetimes. Diffusion can occur across such a layer, but once a particle diffuses to the edges, it can go no further. It then waits until, purely by chance, another layer forms on top of the particle, but displaced vertically relative to the previous layer. Diffusion may then occur across this new layer, until the particle reaches an upper or lower edge (depending on the direction of diffusion) or until the layer dissipates. Thus, the rate of vertical diffusion over scales deeper than the typical layer depths depends on factors including the depth of the layers, and the frequency of occurrence of the layers, (as 

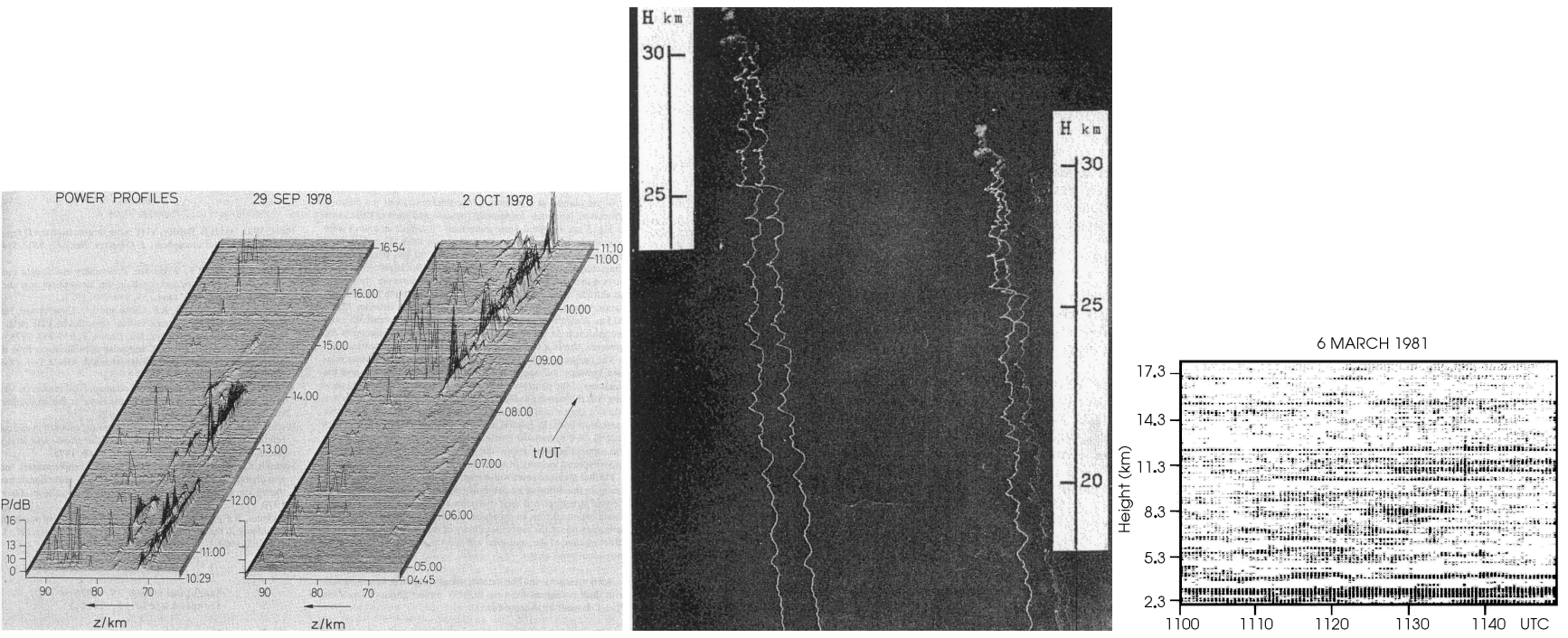

Fig. 1. (a) Illustration of stratification of radar echoes at VHF (from Czechowsky et al., 1979). (b) Evidence of horizontal stratification of atmospheric winds using in situ techniques (smoke trails). (c) Stratification of persistent thin sheets in the troposphere and lower stratosphere (from Hocking and Röttger, 1983).

well as the strength of turbulence within the layers). More details about this process can be found in Dewan (1981), Woodman and Rastogi (1984), and Hocking (1991, 1999), among others. This understanding of turbulent diffusion has been developed, in part, due to the contributions of radar observations which offer one of the best tools to implement calculations of diffusion in this manner, as described by Woodman and Rastogi (1984).

\section{Intermediate-scale studies ( $\sim 1 \mathrm{~m}-100 \mathrm{~m})$}

While radars have made important contributions to our understanding of the large-scale structure of turbulence, they have also made contributions at smaller scales. Interestingly, this has been a difficult challenge, since it often involves examination of scales which are less than the radar pulse length. Various specialized techniques must be employed in order for scales of the order of $10 \mathrm{~m}$ or less to be resolved, including techniques like frequency- domain interferometry (FDI), which is a simplified FM/CW radar techique, as well as more sophisticated deconvolution procedures.

It is even possible to look at metre-size scales if a radar has a sufficiently high frequency, with a correspondingly short wavelength. At these higher frequencies, large bandwidths are possible, thereby allowing very good range resolution. For example, powerful FM-CW radars have been used to obtain high resolution studies of turbulent events in the atmospheric boundary layer (e.g. Gossard et al., 1970, 1978; Eaton et al., 1995). Figures 3 and 4 show examples of such observations taken from Eaton et al., (1995); these figures are very informative, and show excellent resolution. The radar used to create these pictures works at a much higher frequency than the ones under general discussion in this review, but we have already indicated that we will utilize data from other frequencies if it helps us to understand the processes which are observed within the MF, HF and VHF bands. These images clearly help us visualize these smaller scales. However, such images are generally only possible in the lowest few kilometres of the atmosphere, since the radars used for these studies receive very little backscatter from higher altitudes. Hence, there is also the possibility that some of these results are specific to the atmospheric boundary layer and lower levels of the troposphere. Nevertheless, there are still some features which we can find useful.

First, the figures do demonstrate that "cat's-eye" structures are not uncommon in the atmosphere as a precursor to turbulence breakdown (Fig. 3), and such structures are often associated with Kelvin-Helmholtz $(\mathrm{K}-\mathrm{H})$ billows. However, some caution is needed with regard to this observation. It needs to be kept in mind that K-H need not be the only mechanism responsible for the formation of cat's-eye billows; Smyth and Peltier (1989) have indicated that other mechanisms, such as the Holmboe instability, can also generate such structures. Indeed, K-H instabilites are only dominant in weakly stratified flows, whereas the Holmboe instability is more likely in strongly stratified flows.

Second, we see from Fig. 4 that there can be occasions when other mechanisms of breakdown can be responsible for the turbulence; the formation of cat's-eye structures is only one possible mechanism. Cat's-eye structures are visually impressive, so they tend to dominate the literature, but it is not clear whether they are in fact the main mode of turbulence breakdown at all.

Thus, while high-resolution studies of the type shown in Figs. 3 and 4 are very useful, it is still not clear how relevant these boundary layer studies are to understanding turbulence generation in the stratosphere and mesosphere. Cho et al. (1996) have made higher level, high-resolution stud- 

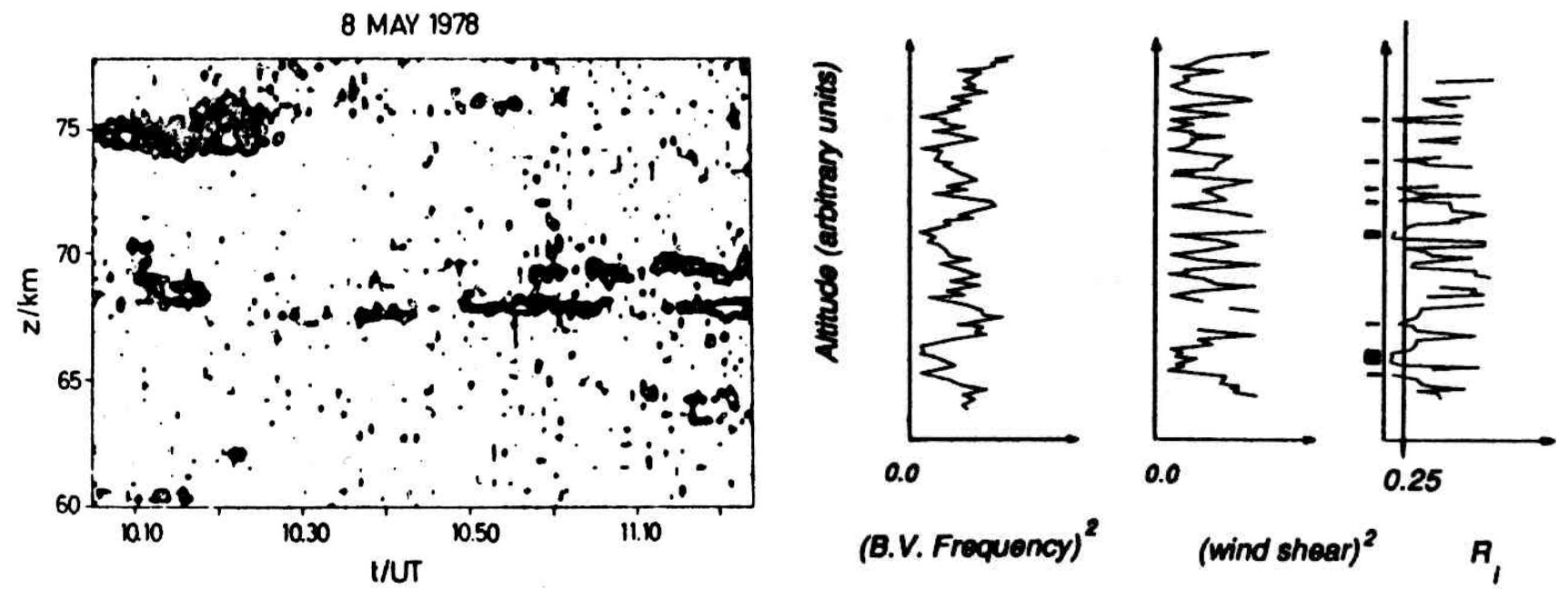

(B.V. Frequency)
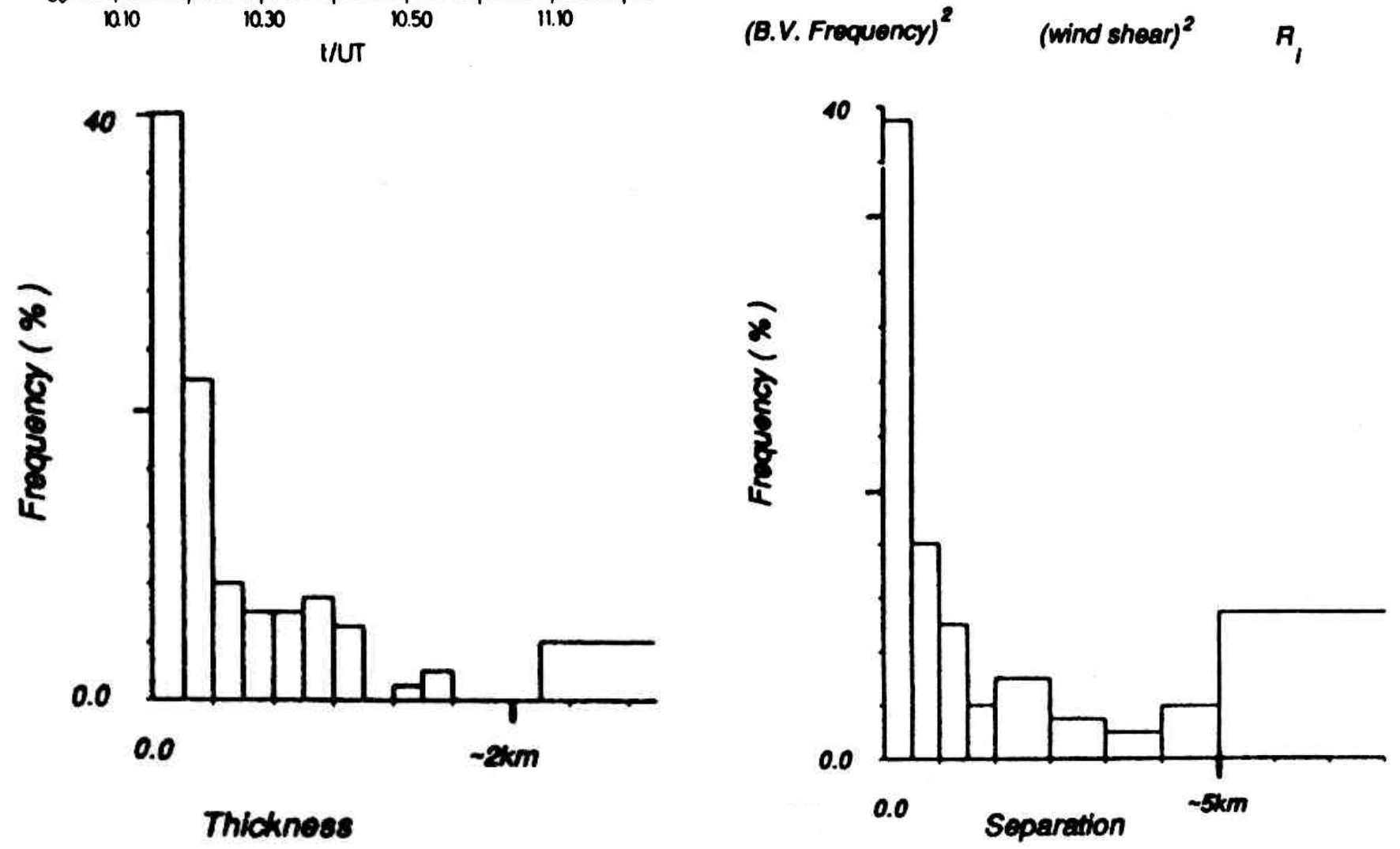

Fig. 2. (a) Another example of stratified radar echoes. (b) Typical profiles of Brunt-Vaisala frequency, wind shears, and resultant Richardson numbers, for a sample gravity wave field. (c) Distribution of the thicknesses of layers of turbulence expected in the stratosphere. Scales are only representative, and somewhat larger in the mesosphere than in the stratosphere. (d) Histogram of expected layer spacings. These figures have been adapted from Hocking (1991). 


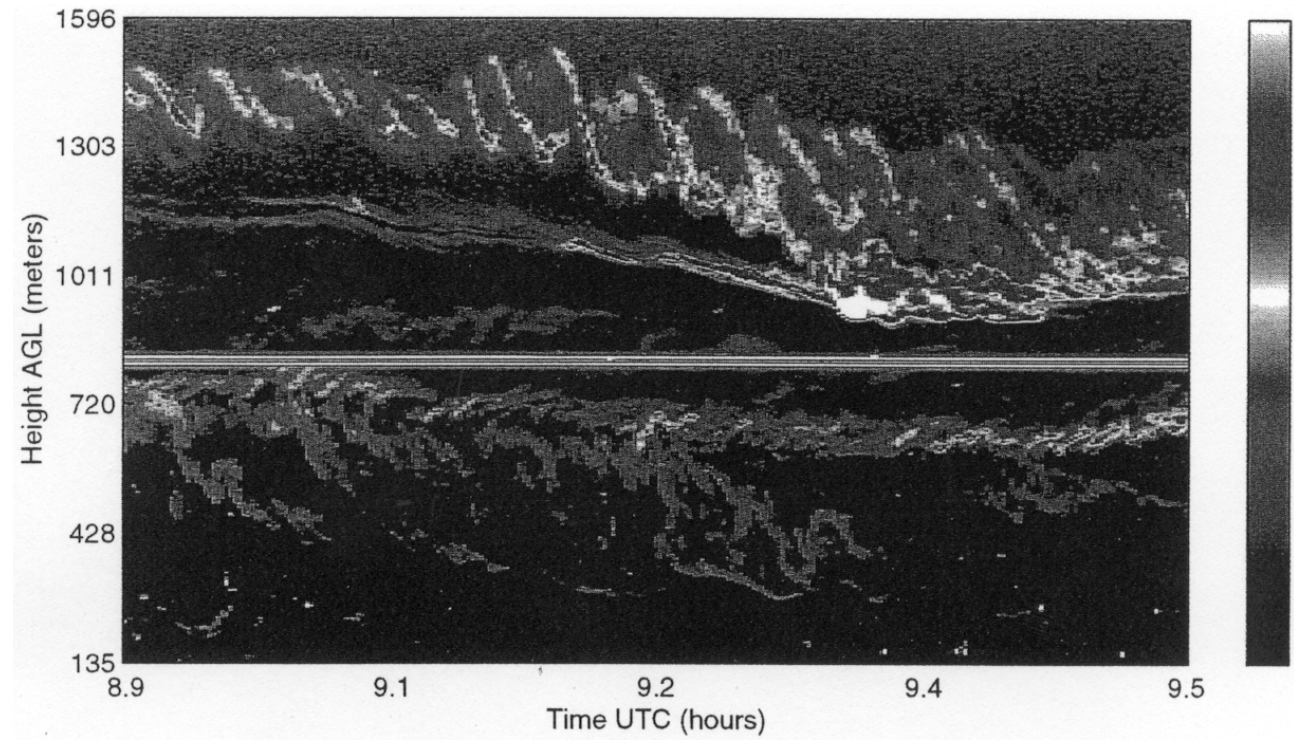

Fig. 3. FMCW radar images of low level turbulence, in this case, showing Kelvin-Helmholtz billows (from Eaton et al., 1995).

ies using astronomical telescopes working at a frequency of $2320 \mathrm{MHz}$, and Woodman (1980) has used the Arecibo 2380 $\mathrm{MHz}$ radio telescope to perform high-resolution studies of stratospheric layers; such studies are rare. Nevertheless, we recognize that radars are at least able to make some useful studies of turbulence breakdown at high-resolution, and have already been very useful in this regard. More of such studies are very much needed. It is especially important to develop statistics about the probabilities of turbulence generation by different mechanisms, free of any preconceived theoretical biases. This remains a topic for future experimental studies.

We now return to the focus of this paper, which is radars operating in the frequency range between $2 \mathrm{MHz}$ and 300 $\mathrm{MHz}$ (MF to VHF). These radars cannot achieve the resolution of FM/CW radars operating in the Gigaherz frequency range (discussed above), but they can achieve a better height coverage. VHF radars can detect signals as high as $90 \mathrm{~km}$ in altitude in the middle atmosphere. These radars are often pulsed, with pulse-lengths in the range of $150 \mathrm{~m}$ to $2 \mathrm{~km}$. Normally these radars cannot resolve structure at scales less than the pulse length. However, there are certain procedures which can be used to improve the resolution under certain circumstances. One example is shown in Fig. 5, which demonstrates the application of deconvolution procedures (Röttger and Schmidt, 1979).

Reid et al. (1987) have shown high-resolution studies of cat's-eye like structures in the mesosphere using a VHF radar. Other procedures also exist to achieve better spatial resolution, such as FDI (Frequency Domain Interferometry, e.g. Kudeki and Stitt, 1987; Chilson et al., 1996, among others), SDI (Spatial Domain Interferometry, e.g. Pan and Röttger, 1996; Hocking, 1997) and newly developed signal processing methods (e.g. Luce et al., 2000; Palmer et al., 2000). However, it should also be noted that the advantages of improved vertical resolution can be negated if there is not a corresponding improvement in horizontal resolution, and the ideal procedure would be to apply both FDI and SDI simultaneously to produce improvements in both vertical and horizontal resolution (see Röttger et al., 2000).

\section{Small-scale studies (metres and less)}

At scales of metres and less, radars provide even less direct information than at intermediate scales. Nevertheless, these scales are still of great interest. As an example of the types of motions which we would like to observe, Fig. 6 shows streak-photographs of small-scale motions taken in a laboratory setting (in this case, for two-dimensional turbulence, so it may not be entirely representative of small-scale atmospheric turbulence). Eddy motions are clear in both figures, and some ellipticity is evident in some eddies. It is important to know just how representative these pictures are of atmospheric processes at scales of a metre and less. Can radars reveal anything about the motions at these smallest scales? We have seen that FM CW radars can provide information about backscattered or partially reflected powers at scales approaching this resolution, but they have yet to produce velocity maps at such scales. What about VHF and lower frequency radars? Can they provide any information at these scales? In fact, they can, at least indirectly, and one area in which radars have made very important contributions is in the area of anisotropy studies.

To demonstrate the issue at hand here, we show Fig. 7. This figure (adapted from Pao, 1968) shows flow around an object in salt-stratified water, at points close to the object as well as further downstream, and the point to be emphasized here is the way in which the turbulence tends to "striate" and stratify as it dies out. The initial active turbulence is clearly isotropic at the smaller scales, while the turbulence which 


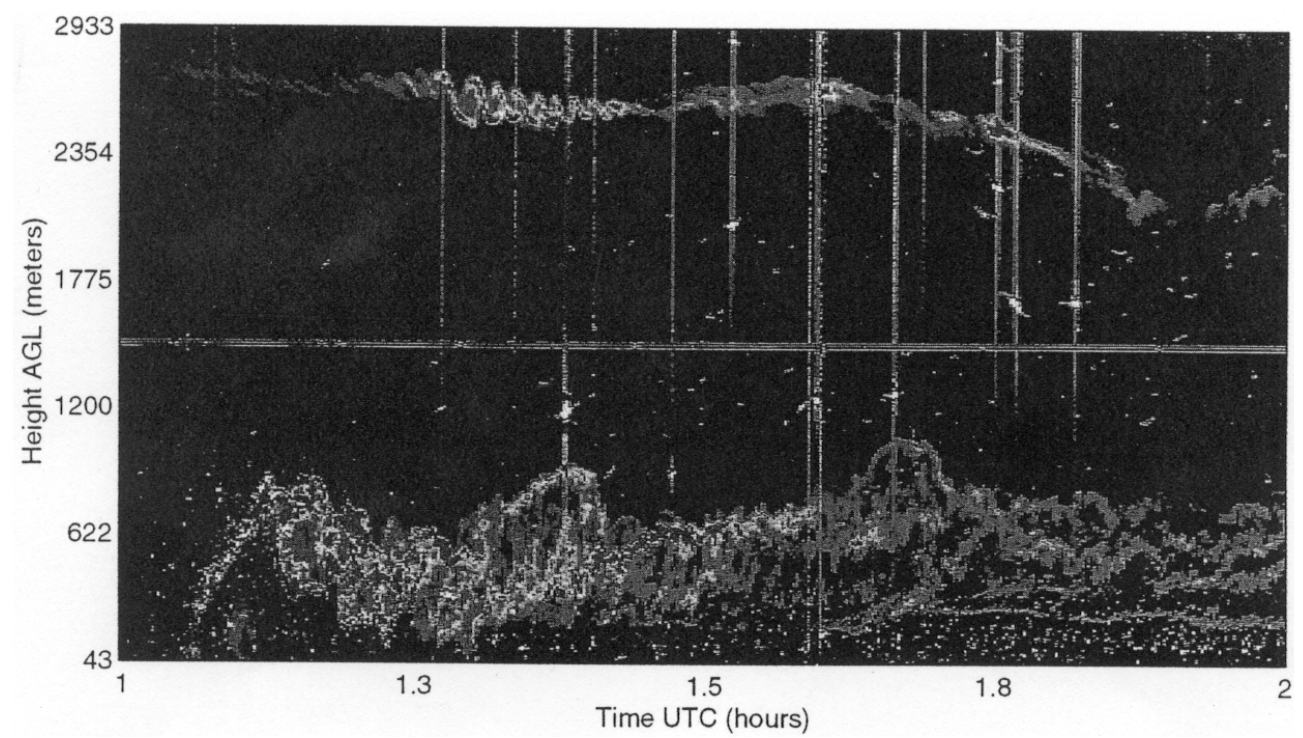

Fig. 4. Another FMCW radar image of low level turbulence, in this case, showing frontal turbulence with no sign of K-H billows (again adapted from Eaton et al., 1995). The entire picture covers a period of one hour in duration, and covers the height range from a 40 metre altitude to $1.8 \mathrm{~km}$ altitude.

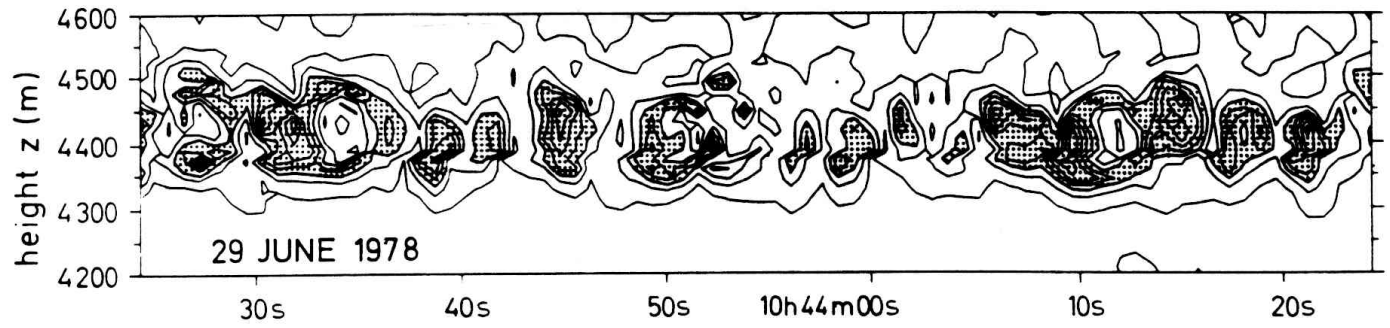

Fig. 5. Results of the application of deconvolution procedures to achieve sub-pulse-length resolution with a VHF radar. Cat's-eye structures are evident.

persists further downstream (which may be either steadystate or decaying) is more anisotropic. Therefore, we ask the question: to what level do we expect atmospheric turbulence to be isotropic? Do the "eddies" shown in Fig. 6 form, and if so, do they exhibit any form of anisotropy, i.e. do they tend to be elongated horizontally relative to their vertical extent? This is an area where radar studies have made substantial contributions.

To understand the contributions of radars in this field, it is necessary to show how "eddy anisotropy" is parameterized; this is illustrated in Fig. 8a. As seen in Fig. 6, velocity trajectories are often elliptical in shape, but individual refractive index "entities" (radio-scatterers) are not always so organized. Even an initially "organized" shape is quickly torn in various directions by the underlying velocity field, so that individual refractive index "entities" within a turbulent region often have stretched and distorted string-like shapes just as one can see different "shapes" and structures within an ordinary water vapour cloud. Hocking and Hamza (1997) have discussed this process in some detail. However, despite the fact that individual entities may be distorted in shape, the spatial correlation function of the refractive index fluctuations does have a broadly elliptical shape with a smoothly varying cross section, where the cross sectional profile is often assumed to be Gaussian. In determining the general properties of radar backscatter, it is often this correlation function which is most important (e.g. see Doviak and Zrnic, 1984). Thus, we often represent atmospheric radio-wave scatterers as if they were ellipsoids, since the spatial correlation function of a field of ellipsoidal scatterers with Gaussian cross section is also an ellipsoidal function with Gaussian cross section. Thus, we can represent the refractive index fluctuations in a turbulent field as if it were comprised of such structures, as shown in Fig. 8a (also see de Wolfe, 1983).

The eddies which are most effective at producing radar backscatter are those with vertical dimensions of the order of 0.1 to 0.5 radar wavelengths (which contain significant Fourier components at the Bragg scale). Larger and smaller structures are very inefficient scatterers. Hence, studies of the degree of anisotropy of backscatter allow us to investigate the structure of the turbulence at scales which are below the radar wavelength, and comparable with the Bragg scales (e.g. see Hocking 1987a). When we recognize that such anisotropy exists, then it also becomes clear that a mono- 

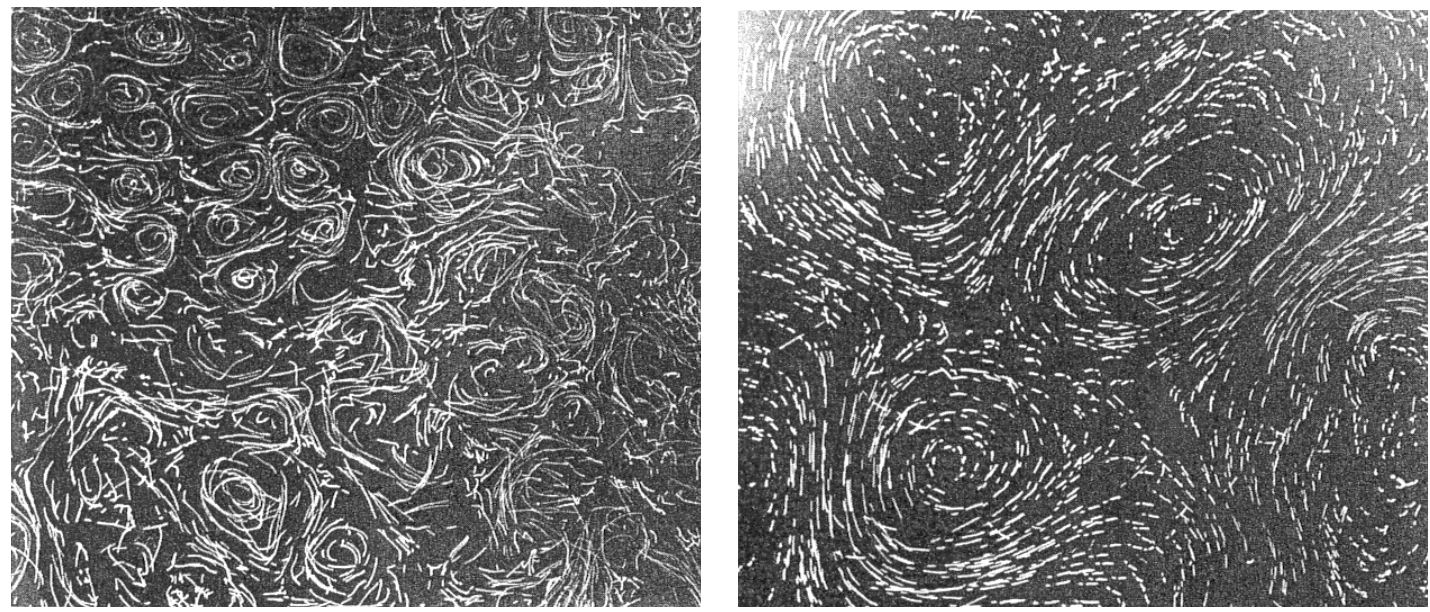

Fig. 6. Streak photography images of small-scale turbulence (in this case, the graphs are for two-dimensional turbulence). The graphs refer to different conditions of stability; from Hopfinger (1987), who adapted it from Maxworthy et al. (1985).

static radar will produce backscattered power that varies as a function of the beam bore direction.

When the radar beam is pointed vertically, backscattered power will be at a maximum, whilst backscattered power decreases with increasing zenith angles as the beam is pointed further and further off vertical. This is very common for radars operating at wavelengths of larger than a few metres with antenna beams pointing at or close to the vertical. Such aspect sensitivity has so far not been detected with radars working at wavelengths of less than about one metre. For scatterers of the type described, the power falls off proportionally to $\exp \left(-\sin ^{2} \theta / \sin ^{2} \theta_{s}\right)$, where $\theta$ is the angle of tilt of the beam from vertical, and $\theta_{s}$ is referred to as the "aspect-sensitivity" parameter. Smaller values of $\theta_{s}$ refer to more anisotropic scatterers, when the ratio $L / h$ (Fig. 8a) is largest. By measuring the power received as a function of angle, it is possible to determine this parameter. Other methods may also be used which involve comparison of signal characteristics recorded with radar beams pointed at different zenithal angles. An example of such measurements is shown in Fig. 8b, in this case for a $2 \mathrm{MHz}$ radar. More extensive discussions of the conversions between $L / h$ and $\theta_{s}$ can be found in Hocking (1987a), Lesicar et al. (1994) and Hocking and Hamza (1997), among others.

A word of warning must be noted here, however. Not all atmospheric radio scatterers take the form of turbulent entities. There are also other, highly structured so-called "specular reflectors" in the atmosphere, which produce "mirrorlike" partial reflections. In calculating $\theta_{s}$, it is necessary to be certain that such specular reflectors do not coexist near the turbulence, thereby contributing a non-turbulent component to the radar signal. The cause of these thin sheets or laminae is still uncertain, although various suggestions have been made (e.g. Hocking et al. 1991). Röttger (1980b) and others have suggested that it would be useful to compare such radar observations with observations of similar structures in the ocean. For an improved understanding of these atmo- spheric structures, high-resolution radar observations, combined with high-resolution in situ measurements are required (e.g. Luce et al., 1995). These special reflectors were first considered as early as almost forty years ago (Beckman and Spizzichino, 1963; Atlas, 1964). Their existence and the fact that the reflection is from a rough or corrugated surface must be recognized. Further discussions about them can be found in Gage (1990), Röttger and Larsen (1990), Hocking et al. (1991), and Hocking (1996), among others. How these corrugated sheets and laminae are generated and how they are related to turbulence remains a challenging question to be solved. We will come back to a consideration of these questions shortly.

It has also been found that these scatterers have an azimuthal asymmetry, and this is also an area of some interest. For example, Hocking (1987b) showed an azimuthal variation of the so-called "Rice parameter" which may indicate preferential stretching of the scatterers according to the wind direction, and Tsuda et al. (1997) also found anisotropy in the backscattered power as a function of azimuth. Nevertheless, considerable extra work is required in this area, but the point remains that radars have a lot to offer in this regard.

Thus, with due care, radars can be used to measure the degree of anisotropy in a turbulent regime. This is an important parameter which cannot be otherwise easily accessed. It also needs to be recognized that the aspect-sensitivity parameter is generally scale-dependent, as described by Hocking and Hamza (1997). Multiple frequency radar studies can help examine this scale dependence.

There is one further feature which needs to be recognized in regard to the turbulence anisotropy, and this is the fact that the degree of anisotropy has long been recognized to be a function of position within the turbulent layer. Several authors have recognized this (e.g. Peltier et al., 1978; Röttger et al., 1981; Hocking, 1985; Woodman and Chu, 1989; Hocking, 1991). Figure 9 shows one such illustration of the expected variation of anisotropy across the layer, with more 


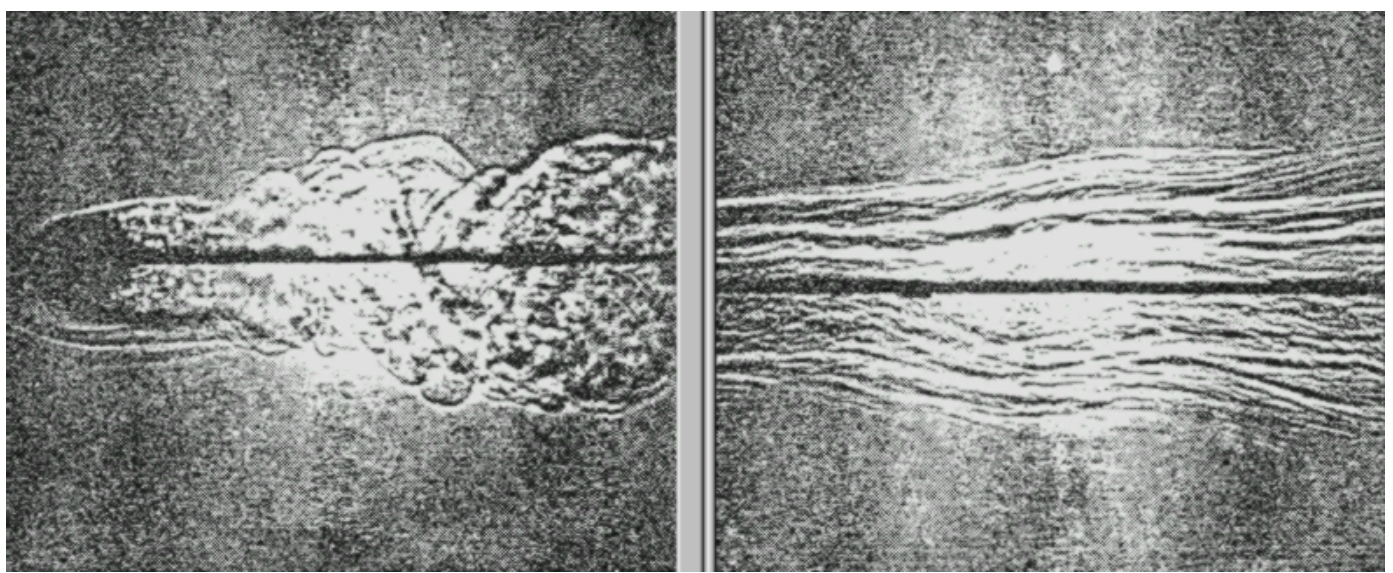

Fig. 7. Photographic images of turbulence generated by a flow around an object (Pao, 1968). The left-hand panel refers to the flow close to the object, and is clearly fairly isotropic at the smaller scales. The right panel shows the flow further downstream, as the turbulence dies out, and stratification is clearly evident.

anisotropic scatterers towards the edges. Other authors have presented similar diagrams (e.g. Röttger 1981; Lesicar and Hocking, 1992; Lesicar et al., 1994). Some of these deliberations have their origin in initial proposals by Bolgiano (1968) about the structure of the atmospheric turbulence (and especially with regard to the edges of the layers). We note that the recent modelling work using super computers (Fritts et al., 1994; Werne and Fritts, 1999; Gibson-Wilde et al., 2000) just confirm these principles which had been developed and have emerged from radar observations over the past decades.

Figure 10 shows the expected structure across a turbulent layer in even greater detail. This graph, adapted from Hocking (1991), combines data from modeling studies (Klaassen and Peltier, 1985a, b), in situ observations (Dalaudier and Sidi, 1987), and knowledge about aspect sensitivity deduced from radar observations. The right-hand profile (solid line) shows the expected mean temperature profile across a typical turbulent layer, as determined by modeling studies (Klaassen and Peltier, 1985a, b). Note, in particular, the relatively sharp edges of the layer, and the near-adiabatic conditions in the middle of the layer. The consequences of this structure are to produce more anisotropic scatterers or specular reflection at the edges, where the background temperature is horizontally stratified but highly fluctuating as a function of height (therefore containing large gradients), and to produce more isotropic scatterers towards the centre, where the temperature profile is closer to adiabatic. This is illustrated by the central part of the figure, which shows anisotropy at the edges and more isotropy towards the centre. The graph on the left shows the wind and temperature fluctuations measured by a balloon passing through such a turbulent layer. Note that the wind fluctuations are similar throughout the layer, but the temperature fluctuations are much larger at the edges, and smaller in the adiabatic portion. The fact that the temperature fluctuations are small near the centre of the layer arise because the background temperature gradient is close to adiabatic. This has important implications for radar scatter as well. We will first discuss why an adiabatic mean temperature gradient produces small temperature fluctuations, and then consider the implications for radar backscatter.

Consider a parcel of air embedded in the atmospheric background, and assume that the parcel and the background are identical in density, temperature, humidity and so forth. Now assume that the parcel is displaced vertically by the turbulence. It undergoes adiabatic changes in temperature and density. At the same time, the temperature of the background air also changes as the height of the parcel changes, but in a manner different to that of the displaced parcel. The change in the background with increasing height depends on the mean background temperature gradient (which need not be adiabatic). The temperature fluctuations measured by a probe will depend on the difference between the temperature of the displaced parcel and the immediate environment. If the mean background temperature gradient is adiabatic, there will be no difference between the temperature of the parcel and that of the background, resulting in zero (or at least small) temperature fluctuations.

Now recall that the refractive index of the air depends upon the temperature, humidity and/or electron density. Just as the temperature fluctuations observed by a probe depend on the difference in temperature between the temperatures of a displaced parcel and its surrounding medium, similarly the radar backscattered signal strength depends on the difference in the refractive index between the displaced parcel of air and the refractive index of the surrounding background air at the same height (e.g. see Hocking 1985 and references therein). If the atmosphere is dry and electron-free (as in the upper troposphere and stratosphere), then the refractive index depends only on the temperature, and so there will be no associated refractive index fluctuations in the case that the mean temperature gradient is adiabatic. Hence, the turbulent layer will be "invisible" to the radar. If a layer exists like the one in Fig. 10, then only the edges of the layer will actually be seen by a radar, giving rise to an apparent "dual layer". Thus, one 


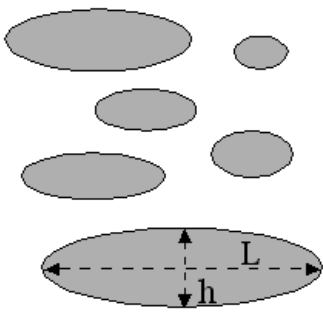

(a)

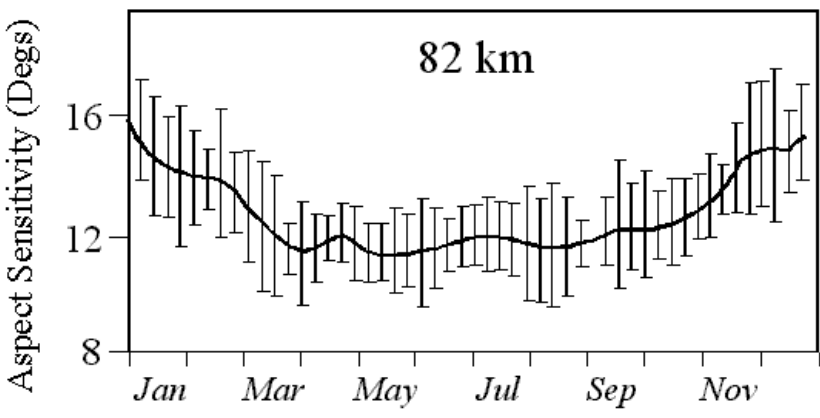

(b)

Month of Year

Fig. 8. (a) Illustration of the representation of atmospheric scatterers within a patch of turbulence. (b) Aspect sensitivity parameter $\theta_{s}$ as a function of the month of the year for a $2 \mathrm{MHz}$ radar detecting scatter from an $82 \mathrm{~km}$ altitude in the mesosphere (from Lesicar and Hocking, 1992).

needs to take care with the interpretation that the strongest scattering layers are the layers of strongest turbulence. Indeed, sometimes the more intense turbulence layers can be the least easily seen with radar. This fact was also recently raised by Gibson-Wilde et al. (2000) using computer simulations, although unfortunately, these authors did not draw a parallel between their observations and the extensive references and types of discussions in the literature considered here.

\section{Specular Reflections}

While we have concentrated so far on the consideration of turbulence as the primary scatter mechanism, we also have commented on the importance of specular partial reflection. This review would not be complete without at least some discussion about these reflectors. Observations of strongly aspect sensitive reflections have been reported many times in the literature, (e.g. Röttger and Liu, 1978; Gage et al., 1978; Hocking, 1979; Fukao et al., 1979; Tsuda et al., 1986; Hocking et al., 1990, 1991; Hooper and Thomas, 1995), to name but a few. Observations of very slow fading times have also been associated with specular reflections.

These studies have been the subject of some controversy. At the one extreme, there have been proposals that specular reflectors are artifacts of the analysis, and all scatter is, in reality, turbulent. Proponents of this approach claim that all anisotropy is due purely to turbulent anisotropy. They cite the fact that in order for specular reflectors to occur, vertical steps in the refractive index of less than a quarter of the radar wavelength are required (e.g. see Hocking and Röttger, 1997), which must be horizontally extended over typically a Fresnel zone in size, and they consider this to be unrealistic. At the other extreme, there have been proposals which claim that almost all scatter is, in fact, a reflection from specular reflectors, and that variations in the parameter $\theta_{s}$ arise simply due to the fact that the specular reflectors have varying degrees of tilt. A superposition of specular reflectors with various tilt angles will produce a corrugated surface. Pos- sible mechanisms to explain these specular reflectors have been given by various authors. Bolgiano (1968) considered that they might be sharp edges to turbulent layers, Van Zandt and Vincent (1983) suggested the possibility that they were short wavelength gravity waves, Hocking et al. (1991), (with an errata in Hocking, 1996) considered the importance of socalled viscosity waves, and Klostermeyer (1992) discussed the possibility that gravity- wave breakdown does not lead to chaotic motion, but leads rather to small-scale organized motions via parametric instability. In this last model, the motion remains organized down to very small scales, but the superposition of the many different small-scale waves produced in this way gave the appearance of a "turbulent" region.

Experimentally, there have been a wide variety of interpretations of radar backscatter characteristics. Tsuda et al. (1997) attribute their observed azimuthal anisotropy entirely to "the characteristics of specular reflection rather than the effects of localized turbulent scattering". Cho and Röttger (1997) discussed whether that polar mesospheric summer echoes contain substantial contributions from specular reflections. Other observers have attributed all their observations to turbulent scatter. In an attempt to delimit turbulent and specular reflections, Hocking and Hamza (1997) developed a formalism which related the extreme values of $\theta_{s}$ to geophysical parameters like the Richardson number. It was possible to place lower limits on the expected values for $\theta_{s}$ under the assumption that the scatter was due to turbulence. Any smaller values must have been the result of specular reflection. Unfortunately, the process does not work in reverse. In principle, specular reflectors can have any value of $\theta_{s}$; larger values are permitted simply by allowing a greater variance in the degree of tilting or surface roughness.

Hence, it must be kept in mind when considering the points raised in this paper that there is a possibility that turbulent scatter may not be the dominant scatter mechanism for VHF, HF and MF radars. Our own opinion is that both types of scatter exist, and care must be taken to determine which applies. Hocking (1987a) has shown examples of the simultaneous occurrence of specular reflectors and turbulent scatter at MF, so it is our belief that the mechanisms of backscat- 


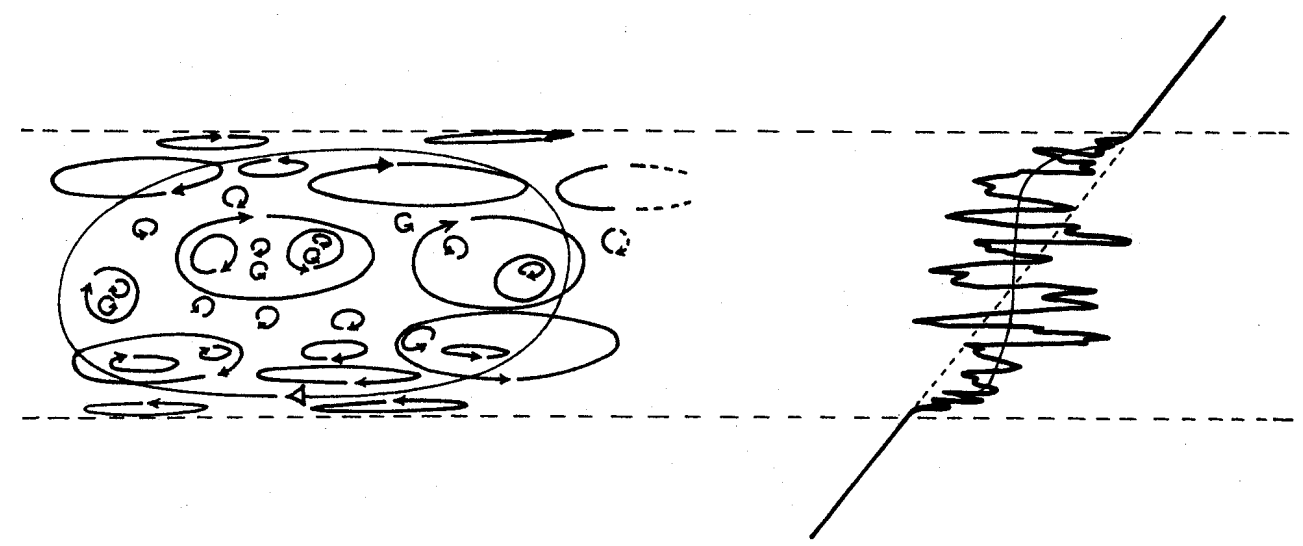

Fig. 9. Illustration from Woodman and Chu (1989) showing a proposal for the structure of radio-wave scatterers within a turbulent layer. More anisotropic scatterers are expected near the top and bottom of the layer. Several other similar proposals appear in various papers in the literature (see text for details).

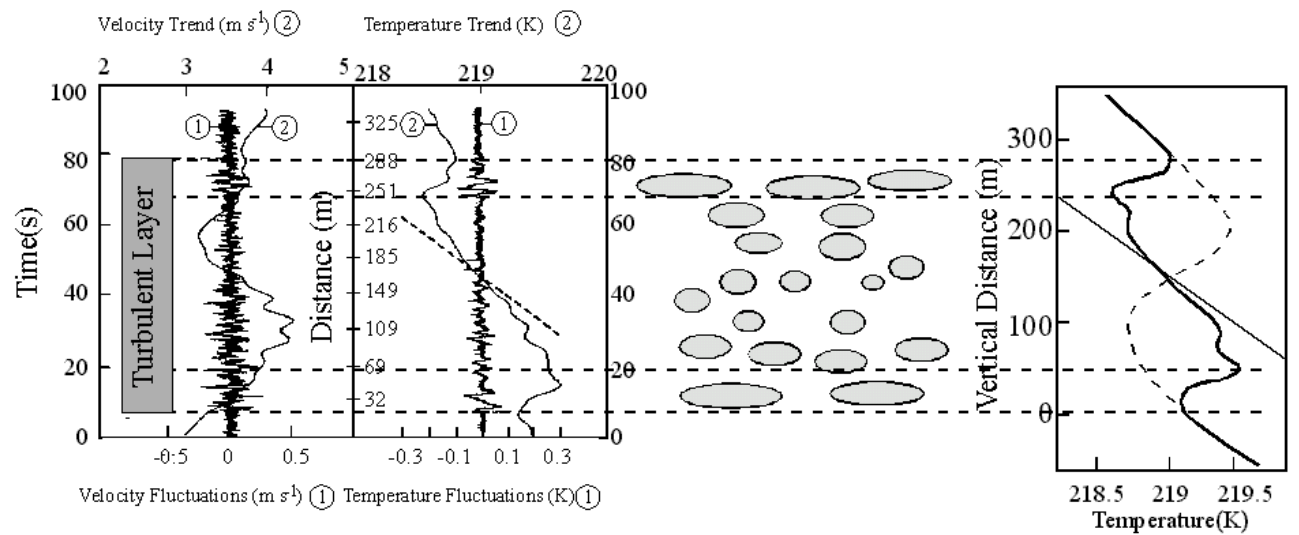

Fig. 10. Expected behaviour of velocity, temperature, and refractive index inhomogeneities within a turbulent layer. In the left-hand two graphs, the smoother lines represent the mean wind and temperature profiles (labelled (2)), while the more rapidly varying lines passing up through the centre (labelled (1)) represent fluctuations of the appropriate parameter about the mean. In the right-hand graph, the broken line represents an intitial temperature profile and the solid one represents the profile after the turbulence is established (from modeling studies). Note that the vertical scalings are slightly different in the left- and right-hand profiles, since each comes from a different source. We have adjusted the scalings so that the temperature profiles look similar. In between these graphs we show the shapes of "average" eddies as a function of position within the layer, demonstrating greater anisotropy at the top and bottom of the layer. See text for other details.

ter are neither exclusively turbulent, nor exclusively specular. The comments we have made within this article refer only to the cases in which turbulent backscatter is the dominant mechanism responsible for the returned signal.

\section{Conclusions}

By utilizing radar data, in situ data, and early modeling studies, it has been possible to deduce significant information about the large- and medium-scale structure, and the internal structure of turbulent layers in the atmosphere. Radars are especially useful for displaying the large-scale morphology of turbulence, and by using sophisticated processing techniques, they can also be useful in describing the mediumand small-scale structures. They are especially powerful for studying the anisotropy and morphology at different scales of turbulent atmospheric regions. This paper has concentrated especially on the contribution which radars have made to these determinations and how they can continue to contribute further. The combination of in situ and modeling techniques will certainly supplement these investigations.

Acknowledgements. Topical Editor J.-P. Duvel thanks T. E. Van Zandt and another Referee for their help in evaluating this paper.

\section{References}

Atlas, D., Advances in Radar Meteorology, in Adv. Geophys.,10, eds. Landsberg and Miegham, Academic Press, 317-478, 1964.

Beckman, P. and Spizzichino, A., The scattering of electromagnetic waves from rough surfaces, Pergamon Press, Oxford, pp. 503, 1963.

Blamont, J. E. and Barat, J., Dynamical structure of the atmosphere 
between 80 and $120 \mathrm{~km}$, from "Aurora and Airglow", ed. B. M. McCorrmac, p. 159, Reinhold Publ. Co., 1967.

Bolgiano, R., The general theory of turbulence. Turbulence in the atmosphere, in Wind and Turbulence in Stratosphere, Mesosphere and Ionosphere, ed. K. Rawer, North Holland Publ., Amsterdam, 371-400, 1968.

Bondarev, V. D., Lebedinets, V. N., Prorok, V. P., and Sarkisyan, A. M., Investigation of a fine layered wind field structure in the atratosphere by the artificial luminous cloud method, Adv. Space Res., 12(10), 161-163, 1992.

Chilson, P.B. and Schmidt, G., Implementation of frequency domain interferometry at the SOUSY VHF radar: first results, Radio Sci., 31, 263-272, 1996.

Cho, J. Y. N., Jurgens, R. F., and Slade, M. A., High-resolution stratospheric dynamics measurements with the NASA/JPL Goldstone solar system radar, Geophys. Res. Letts., 23, 1909-1912, 1996.

Cho, J. Y. N. and Röttger, J., An updated review of polar mesosphere summer echoes: Observation, theory, and their relationship to noctilucent clouds and subvisible aerosols, J. Geophys. Res., 102, 2001-2020, 1997.

Czechowsky, P., Ruester, R., and Schmidt, G., Variations of mesospheric structure in different seasons, Geophys. Res. Letts., 6, 459-462, 1979.

Dalaudier F. and Sidi, C., Evidence and interpretation of a spectral gap in the turbulent atmospheric temperature spectra, J. Atmos. Sci., 44, 3121-3126, 1987.

Desaubies, Y. and Smith, W. K., Statistics of Richardson number and instability in oceanic internal waves, J. Phys. Oceanography, $12,1245-1259,1982$.

Dewan, E. M., Turbulent vertical transport due to thin intermittent mixing layers in the stratosphere and other stable fluids, Science, 211, 1041-1042, 1981.

Doviak, R. J. and Zrnic, D. S., Reflection and scatter formula for anisotropically turbulent air, Radio Sci., 19, 325-336, 1984.

Eaton, F. D., McLaughlin, S. A., and Hines, J. R., A new frequencymodulated continuous wave radar for studying planetary boundary layer morphology, Radio Sci., 30, 75-88, 1995.

Fairall, C. W., White, A. B., and Thomson, D. W., A stochastic model of gravity-wave-induced clear-air turbulence, J. Atmos. Sci., 48, 1771-1790, 1991.

Fritts, D. C., Isler, J. R., and Andreassen, O., Gravity wave breaking in two and three dimensions, 2, Three dimensional evolution and instability structure, J. Geophys. Res., 99, 8109-8123, 1994.

Fukao, S., Sato, T., Kato, S., Harper, R. M., Woodman, R. F., and Gordon, W. E., Mesospheric winds and waves over Jicamarca on 23-24 May 1974, J. Geophys. Res., 84, 4379-4386, 1979.

Gage, K. S. and Green, J. L., Evidence for specular reflection from monostatic VHF radar observations of the stratosphere, Radio Sci., 13, 991-1001, 1978

Gage, K. S., Radar observations of the free atmosphere: Structure and dynamics, in Radar in Meteorology; Battan Memorial and $40^{\text {th }}$ Anniversary Radar Meteorology Conference, edited by D. Atlas, pp 534-565, Amer. Meteorol. Soc., Boston, Mass., 1990.

Gibson-Wilde, D., Werne, J., Fritts, D., and Hill, R., Direct numerical simulation of VHF radar measurements of turbulence in the mesosphere, Radio Sci., 35, 783-798, 2000.

Gossard, E. E., Richter, J. H., and Atlas, D., Internal waves in the atmosphere from high-resolution radar measurements, J. Geophys. Res., 75, 3523-3536, 1970

Gossard, E. E., Chadwick, R. B., Moran, K. P., Strauch, R. G., Morrison, G. E., and Campbell, W. C., Observation of winds in the clear air using an FM-CW Doppler radar, Radio Sci., 13, 285$289,1978$.

Hines, C. O., The saturation of gravity waves in the middle atmosphere. Part I: Critique of linear instability theory, J. Atmos. Sci., 48, 1348-1359, 1991a.

Hines, C. O., The saturation of gravity waves in the middle atmosphere. Part II: Development of Doppler-spread theory, J. Atmos. Sci., 48, 1360-1379, 1991b.

Hines, C. O., The saturation of gravity waves in the middle atmosphere. Part III: Formation of the Turbopause and the turbulence layers beneath it, J. Atmos. Sci., 48, 1380-1385, 1991c.

Hocking, W. K., Angular and temporal characteristics of partial reflections from the D-region of the ionosphere, J. Geophys. Res., 64, 845-851, 1979.

Hocking, W. K. and Röttger, J., Pulse length dependence of radar signal strengths for Fresnel backscatter, Radio Sci., 18, 1312$1324,1983$.

Hocking, W. K., Measurement of turbulent energy dissipation rates in the middle atmosphere by radar techniques: a review, Radio Sci., 20, 1403-1422, 1985.

Hocking, W. K., Radar studies of small scale structure in the upper middle atmosphere and lower ionosphere, Adv. Space Res., 7(10), 327-338, 1987a.

Hocking, W. K., Reduction of the effects of non-stationarity in studies of amplitude statistics of radio wave backscatter, J. Atmos. Terr. Phys., 49, 1119-1131, 1987b.

Hocking W. K., Fukao, S., Tsuda, T., Yamamoto, M., Sato, T., and Kato, S., Aspect sensitivity of stratospheric VHF radiowave scatterers, particularly above $15 \mathrm{~km}$ altitude, Radio Sci., 25, 613$627,1990$.

Hocking, W. K., The effects of middle atmosphere turbulence on coupling between atmospheric regions, J. Geomag. Geoelectr. 43, Suppl., 621-636, 1991

Hocking, W. K., Fukao, S., Yamamoto, M., Tsuda, T., and Kato, S., Viscosity waves and thermal-conduction waves as a cause of "specular" reflectors in radar studies of the atmosphere, Radio Sci., 26, 1281-1303, 1991.

Hocking, W. K., Some new perspectives on viscosity and thermal conduction waves as a cause of "specular" reflectors in radar studies of the atmosphere, STEP Handbook, Proceedings of the Seventh Workshop on Technical and Scientific Aspects of MST Radar, Hilton Head Island S. C., USA, 7-11 November 1995, ed. B. Edwards, 82-85, 1996.

Hocking, W. K., Recent advances in radar instrumentation and techniques for studies of the mesosphere, stratosphere, and troposphere, Radio Sci., 2241-2270, 1997.

Hocking, W. K. and Mu, K. L., Upper and Middle Tropospheric KineticEnergy Dissipation Rates from Measurements of $C_{n}^{2}-\mathrm{Re}-$ view of Theories, in situ Investigations, and experimental Studies using the Buckland Park Atmospheric Radar in Australia, J. Atmos. Terr. Phys, 59, 1779-1803, 1997.

Hocking, W. K. and Hamza, A. M., A Quantitative measure of the degree of anisotropy of turbulence in terms of atmospheric parameters, with particular relevance to radar studies, J. Atmos. Solar Terr. Phys., 59, 1011-1020, 1997.

Hocking, W. K. and Röttger, J., Studies of polar mesosphere summer echoes over EISCAT using calibrated signal strengths and statistical parameters, Radio Sci., 32, 1425-1444, 1997.

Hocking, W. K., The dynamical parameters of turbulence theory as they apply to middle atmosphere studies, Earth Planets Space, $51,525-541,1999$

Hooper, D. and Thomas, L., Aspect sensitivity of VHF scatterers in 
the troposphere and stratosphere from comparisons of powers in off-vertical beams, J. Atmos. Terr. Phys., 57, 655-663, 1995.

Hopfinger, E. J., Turbulence in stratified fluids: a review, J. Geophys. Res., 92, 5287-5303, 1987.

Klaassen, G. P. and Peltier, W. R., The onset of turbulence in finiteamplitude Kelvin-Helmholtz billows, J. Fluid Mech., 155, 1-35, 1985a.

Klaassen, G. P. and Peltier, W. R., Evolution of finite amplitude Kelvin-Helmholtz billows in two spatial dimensions, J. Atmos. Sci., 42, 1321-1339, 1985b.

Klostermeyer, J., The formation of layered structures by parametric instabilityof finite-amplitude gravity waves, Adv. Space Res., 12(10), 203-205, 1992.

Kudeki, E. and Stitt, G. R., Frequency domain interferometry: a high resolution radar technique for studies of atmospheric turbulence, Geophys. Res. Lett., 14, 198-201, 1987.

Larsen, M. F. and Röttger, J., VHF and UHF Doppler radars as tools for synoptic research, Bull. Amer. Meteorol. Soc., 63, 996-1008, 1982.

Lesicar, D. and Hocking, W. K., Studies of seasonal behaviour of the shape of mesospheric scatterers using a $1.98 \mathrm{MHz}$ radar, J. Atmos. Terr. Phys., 54, 295-309, 1992.

Lesicar, D., Hocking, W. K., and Vincent, R. A., Comparative studies of scatterers observed by MF radars in the Southern Hemisphere mesosphere, J. Atmos. Terr. Phys., 56, 581-591, 1994.

Luce, H., Crochet, M., Delaudier, F., and Sidi, C., Interpretation of VHF ST radar vertical echoes from in situ temperature sheet observations, Radio Sci., 30, 1002-1025, 1995.

Luce, H., Yamamoto, M., Fukao, S., Helal, D., and Crochet, M., A frequency domain interferometric imaging (FII) technique based on high resolution methods, Ninth International Workshop on Technical and Scientific Aspects of MST Radar - MST9 combined with COST-76 Final Profiler Workshop, Toulouse, France, 13-18 March 2000.

Maxworthy, T., Caperan, Ph., and Spedding, G. R., Twodimensional turbulence and vortex dynamics in a stratified fluid, Third International Symposium on Density-Stratified Flows, Caltech, Pasadena, USA, 1985.

Palmer, R. D., Chilson, P. B., Muschinski, A., Schmidt, G., Yu, T.-Y., and Steinhagen, H., Range imaging using frequency diversity: theory and application, Ninth International Workshop on Technical and Scientific Aspects of MST Radar - MST9 combined with COST-76 Final Profiler Workshop, Toulouse, France, 13-18 March 2000.

Pan, C.-J. and Röttger, J., Structures of polar mesosphere summer echoes observed with the EISCAT VHF radar in the interferometer mode, in Proceedings of the $7^{\text {th }}$ Workshop on Technical and Scientific Aspects of MST Radar, edited by B. Edwards, pp. 252255, STEP Hnadbook, Natl. Oceanic and Atmos. Admin., Boulder, Colo., 1996.

Pao, Y.-H., Document D1-82-0959, Boeing Scientific Laboratories, 1968.

Peltier, W. R., Halle, J., and Clarke, T. L., The evolution of finiteamplitude Kelvin-Helmholtz billows, Geophys. Astrophys. Fluid Dyn., 10, 53-87, 1978.

Reid, I. M., Ruester, R., and Schmidt, G., VHF radar observations of Cat's-eye-like structures at mesospheric heights, Nature, 327, 43-45, 1987.

Röttger, J. and Liu, C. H., Partial reflection and scattering of VHF radar signals from the clear atmosphere, Geophys. Res. Lett., 5, 357-360, 1978.
Röttger, J. and Schmidt, G., High-resolution VHF radar soundings of the troposphere and stratosphere, IEEE Trans. Geosci. Electron., GE-17, 182-189, 1979.

Röttger, J., Reflection and scattering of VHF radar signals from atmospheric refractivity structures, Radio Sci., 15, 259-276, 1980a.

Röttger, J., Structure and dynamics of the stratosphere and mesosphere revealed by VHF radar investigations, Pure Appl. Geophys., 118, 494-527, 1980b.

Röttger, J., The dynamics of stratospheric and mesospheric fine structure investigated with an MST VHF radar, Middle Atmosphere Program Handbook, ed. S. K. Avery, SCOSTEP Secretariat, University of Illionois, Vol. 2, pp. 341-350, 1981.

Röttger, J., Chzechowsky, P., and Schmidt, G., First low-power VHF radar observations of tropospheric and mesospheric winds and turbulence at the Arecibo Observatory, J. Atmos. Terr. Phys., 43, 789-800, 1981.

Röttger, J. and Larsen, M. F., UHF/VHF radar techniques for atmospheric research and wind profiler applications, in Radar in Meteorology; Battan Memorial and 40th Anniversary Radar Meteorology Conference, edited by D. Atlas, pp 235-281, Amer. Meteorol. Soc., Boston, Mass., 1990.

Röttger, J., Luce, H., Yamamoto, M., Fukao, S., Liu, C. H., Pan, C. J., Su, S. Y., and $\mathrm{Wu}, \mathrm{C} . \mathrm{H}$., Combined high-time resolution SDI-FDI experiments with VHF radars, Ninth International Workshop on Technical and Scientific Aspects of MST Radar - MST9 combined with COST-76 Final Profiler Workshop, Toulouse, France, 13-18 March 2000.

Sahr, J. D. and Fejer, B. G., Auroral electrojet plasmairregulararity theory and experiment: A critical review of present understanding and future directions, J. Geophys. Res., 101, 26893-26909, 1996.

Smyth, W. D. and Peltier, W. R., The transition between KelvinHelmholtz and Holmboe instability: an investigation of the overreflection hypothesis, J. Atmos. Sci., 46, 3698, 1989.

Tsuda T., Sato, T., Hirose, K., Fukao, S., and Kato, S., MU radar observations of the aspect sensitivity of backscattered VHF echo power in the troposphere and lower stratosphere, Radio Sci., 21, 971-980, 1986.

Tsuda, T., Gordon, W. E., and Saito, H., Azimuth angle variations of specular reflection echoes in the lower atmosphere observed with the MU radar, J. Atmos. Solar-Terr. Phys., 59, 777-784, 1997.

Van Zandt, T. E., A universal spectrum of buoyancy waves in the atmosphere, Geophys. Res. Lett., 9, 575-578, 1982.

Van Zandt, T. E. and Vincent, R. A., Is VHF Fresnel reflectivity due to low frequency buoyancy waves?, Handbook for MAP, vol. 9 , pp 78-80, SCOSTEP secretariat, University of Illinois, Urbana, 1983.

Werne, J. and Fritts, D. C., Stratifed shear turbulence: Evolution and statistics, Geophys. Res. Lett., 26, 439-442, 1999.

de Wolfe, D. A., A random-morion model of fluctuations in a nearly transparent medium, Radio Sci., 18, 128-142, 1983.

Woodman, R. F., High-altitude-resolution stratospheric measurements with the Arecibo 2380-MHz radar, Radio Sci., 15, 423430, 1980.

Woodman, R. F. and Rastogi, P. K., Evaluation of effective eddy diffusive coefficients using radar observations of turbulence in the stratosphere, Geophys. Res. Lett., 11, 243-246, 1984.

Woodman, R. F. and Chu, Y.-H., Aspect sensitivity measurements of VHF backscatter made with the Chung-Li radar: plausible mechanisms, Radio Sci., 24, 113-125, 1989. 